\title{
Anti-angiogenic Effect of Ginsenoside Rh2 by Downregulation of VEGF in a Zebrafish Model
}

\author{
MA Liman', QI Yongxiao" ${ }^{1}$, Wang Wenji ${ }^{2 *}$ and Zhong Qianyi ${ }^{3 *}$ \\ ${ }^{1}$ School of Medicine, Taizhou University, Taizhou, Zhejiang 318000, P.R. China. \\ ${ }^{2}$ School of Life Science, Taizhou University, Taizhou, Zhejiang 318000, P.R. China. \\ ${ }^{3}$ Taizhou Central Hospital, Taizhou, Zhejiang 318000, P.R. China.
}

\begin{abstract}
A B S T R A C T
Angiogenesis, the formation of new blood vessels, is tightly regulated in the normal organism. Excessive angiogenesis can have detrimental consequences in various processes, including promoting uncontrolled tumor growth. Inhibiting angiogenesis using natural compounds is an important focus in developing new cancer treatment strategies. Ginsenoside Rh2 (G-Rh2), a natural product derived from red ginseng, has been reported to have therapeutic effects on some tumors, but its antiangiogenic effects in zebrafish has not been evaluated. Here we investigated the antiangiogenic of G-Rh2 using the zebrafish angiogenesis model. Morphological observations of the G-Rh2-treated wild-type embryos were performed to evaluate the toxicity of the compound. $\operatorname{Tg}(f i l-E G F P)$ transgenic zebrafish with fluorescent blood vessels were studied to evaluate vascular development defects. G-Rh2 treatment up to $84.85 \mu \mathrm{M}$ did not induce morphological defects in zebrafish. We observed suppression effect of angiogenesis by G-Rh2 in inhibiting intersegmental vessel formation from 30 to $84.85 \mu \mathrm{M}$ in a dose-dependent manner. Moreover, quantitative real-time PCR revealed that G-Rh2 suppressed vascular endothelial growth factor (vegf) mRNA expression but not mRNA expression of its receptors $(f t l, k d r)$. Western blot further confirmed that G-Rh2 treatment resulted in reduced Vegf protein expression. Our study suggested that G-Rh2 may exert anti-angiogenic activity by downregulation of Vegf in zebrafish embryos, thus indicating its role as a potential therapeutic agent against cancer.
\end{abstract}

\begin{tabular}{l} 
Article Information \\
Received 18 September 2019 \\
Revised 12 December 2019 \\
Accepted 20 February 2020 \\
Available online 11 December 2020 \\
Authors' Contribution \\
\hline ML and WW designed the \\
experiments. QY and ZQ raised \\
fish and collected the samples. ML \\
executed the experimental work and \\
worte the article. \\
Key words \\
\hline $\begin{array}{l}\text { Angiogenesis, Ginsenoside Rh2, Vegf, } \\
\text { Zebrafish }\end{array}$
\end{tabular}

\section{INTRODUCTION}

A ngiogenesis is a physiological process in which new blood vessels emerge from preexisting vessels (Wong et al., 2009; Berthod, 2014). Mounting evidence has demonstrated that angiogenesis plays an important role in tumor progression (Kondo et al., 2002; Mantovani, 2010). Therefore, inhibition of the tumor angiogenesis system has become a vital strategy for cancer therapy (Jansen et al., 2004; Dong et al., 2007). The vascular endothelial growth factor (VEGF) is one of the most important activators of angiogenesis and acts by binding to its two tyrosine kinase receptors VEGFR-1 (FLT1) and VEGFR-2 (FLK1, KDR), which are expressed primarily in endothelial cells (Ferrara et al., 2003). Several reports suggested that inhibition of the VEGF pathway is the best-validated therapeutic approach in the cancer treatment (Jain, 2005; Jain et al., 2006; Crawford and Ferrara, 2009). A recombinant humanized anti-VEGF monoclonal antibody (bevacizumab) has been approved by the Food and Drug Administration for first-line

\footnotetext{
* Corresponding author: wangwj@tzc.edu.cn; zhongqy@tzzxyy.com 0030-9923/2021/0001-0111 \$ 9.00/0

Copyright 2021 Zoological Society of Pakistan
}

treatment of several cancers (Yang et al., 2003; Sandler et al., 2006; Saltz et al., 2008). Tyrosine kinase inhibitors targeting the VEGF receptor are also being evaluated in clinical studies (Matulonis et al., 2009; Natale et al., 2009).

Identifying antiangiogenic drugs from natural compounds holds great promise for cancer therapy. Ginseng is a widely used Chinese herb that is associated with a significantly decreased risk of a variety of cancers (Jin et al., 2016). Ginsenoside Rh2 (G-Rh2) is one of the key components isolated from red ginseng with potential bioactivity. G-Rh2 potently induces cellular apoptosis in hepatoma and leukemia Reh cells (Park et al., 2012; Xia et al., 2014). In addition, G-Rh2 exerts anti-cancer activities on tumor growth and metastasis in prostate cancer (Zhang et al., 2015; Huang et al., 2019), colorectal cancer (Han et al., 2016), and other malignant tumors (Nakata et al., 1998; Shi et al., 2014). G-Rh2 inhibited tumor angiogenesis and growth in a Lewis pulmonary carcinoma mouse model (Cui and Qu, 2011). However, whether G-Rh2 exhibits antiangiogenic activity in zebrafish embryos and its underlying mechanism have not been fully investigated.

The zebrafish, Danio rerio, has emerged as an excellent model for studying a wide variety of biological processes (Thisse and Zon, 2002; He et al., 2014). The advantages of studying angiogenesis in zebrafish include 
its external fertilization, early optical clarity and easy genetic manipulation (Santoro, 2014). Moreover, the effects of several anti-angiogenic drugs examined in the zebrafish model were also observed in mammalian systems (Cross et al., 2003). Additionally, the availability of transgenic zebrafish that express enhanced green fluorescent protein (GFP) in the vasculature enables the visualization of vascular defects in live zebrafish embryos (Tran et al., 2007). Previous studies have examined G-Rh2 in various cells and models; however, the effects of G-Rh2 in the zebrafish model have not been explored.

In this study, we investigated the antiangiogenic effect and the potential underlying mechanism of G-Rh2 in transgenic zebrafish embryos.

\section{MATERIALS AND METHODS}

\section{Chemicals and reagents}

We purchased 20(S)-G-Rh2 from Aladdin Co. Ltd., China. Dimethyl sulfoxide (DMSO) was purchased from Sigma Aldrich Chemicals. Pvt. Ltd., USA. G-Rh2 was dissolved in DMSO as $100 \mathrm{mM}$ stock solution. The stock solution was stored at $-20^{\circ} \mathrm{C}$ to maintain stability until use.

Trizol reagent was purchased from Invitrogen (Carlsbad, CA, USA). The PrimeScript RT reagent kit with gDNA Eraser was purchased from Takara (Dalian, China). Zebrafish VEGF antibody, mouse IgG horseradish peroxidase conjugated antibody and $\beta$-actin antibody were purchased from R\&D systems (Minneapolis, MN, USA). The ECL advanced western blotting detection kit was purchased from Advansta (Menlo Park, USA). SU5416 was obtained from Sigma Aldrich (Shanghai, China).

\section{Zebrafish maintenance and embryos collection}

Wild-type (WT) zebrafish and transgenic zebrafish Tg (fli1:EGFP) expressing enhanced GFP in endothelial cells were provided by core facilities at the Zhejiang University School of Medicine. The two zebrafish strains were maintained as described in the Zebrafish Handbook (Westerfield, 2000). In brief, adult zebrafish were maintained in a controlled environment at $28.5^{\circ} \mathrm{C}$ with a $14 \mathrm{~h}: 10 \mathrm{~h}$ light and dark cycle. Fish were fed three times a day with brine shrimp and occasionally with tropical fish food for $5 \mathrm{~min}$. Zebrafish embryos were generated by natural pairwise mating and were raised at $28.5^{\circ} \mathrm{C}$ in embryonic culture medium water $\left(0.75 \mathrm{mM} \mathrm{NaHCO}, 0.5 \mathrm{mM} \mathrm{MgSO}{ }_{4} \cdot 7 \mathrm{H}_{2} \mathrm{O}\right.$, $2 \mathrm{mM} \mathrm{CaCl} \cdot 2 \mathrm{H}_{2} \mathrm{O}$, and $0.08 \mathrm{mM} \mathrm{KCl}$ ).

\section{Drug administration and morphological observation}

At 24 hours post-fertilization (hpf), WT embryos were dechorionated by treatment with protease $1 \mathrm{mg} /$ $\mathrm{mL}$ for $5 \mathrm{~min}$ and washed using embryo water 5-6 times.
Dechorionated embryos were arrayed in a 12-well plate, with approximately 20 embryos per well in $2 \mathrm{~mL}$ embryo water. Each well contained different concentrations of G-Rh2 $(30,42.43,60,84.85,120 \mu \mathrm{mol})$ and plates were incubated for $48 \mathrm{~h}$ at $28.5^{\circ} \mathrm{C}$. Three replicates were performed for every group. Embryos treated with $0.1 \%$ DMSO (solvent) served as a vehicle control. After drug exposure, the survival rate and morphological changes were examined at $12 \mathrm{~h}$ post-treatment (hpt), $24 \mathrm{hpt}$ and 48 hpt. Images were captured using an Olympus Inverted Microscope (Olympus Corporation, Tokyo, Japan). Each experiment was repeated three times.

\section{Assessment of vessel changes in zebrafish embryos by fluorescent microscopy}

At $24 \mathrm{hpf}$, the Tg (fi-1:EGFP) zebrafish embryos were distributed in 12-well plates with 20 embryos per well. Each group was treated with various concentrations of G-Rh2 dissolved in embryo water for $24 \mathrm{~h}$. The intersegmental blood vessels (ISVs) of embryos were observed and imaged at $24 \mathrm{hpt}$ by fluorescent microscopy (Olympus Corporation). DMSO (0.1\%) served as vehicle control and SU5416 (5 $\mu \mathrm{mol})$ as positive control.

\section{Quantitative real-time PCR ( $q P C R$ )}

Zebrafish embryos at $24 \mathrm{hpf}$ were treated with $0.1 \%$ DMSO and various concentration of G-Rh2 for $48 \mathrm{~h}$. At 72 hpf, total RNA was isolated from samples of 30 zebrafish embryos per treatment group using Trizol reagent. RNA $(1 \mu \mathrm{g})$ was reverse transcribed to cDNA using the PrimeScript RT reagent kit with gDNA Eraser according to the manufacturer's instructions. The quality and quantity of RNA were assessed by agarose gel electrophoresis and spectrophotometry. qPCR was performed using the TB Green Premix Ex Taq II (Tli RNaseH Plus) (TaKaRa) on the SteponePlus Real-Time PCR System (Thermo Fisher Scientific). Specific primers for vegf, $f t-1$ and $k d r$ mRNA (vegf-fw/rv, flt 1 -fw/rv, and $k d r$-fw/rv, respectively) were designed and the sequences are shown in Table I. $\beta$-actin was chosen as the reference gene for normalization of gene expressions. Standard reactions $(20 \mu \mathrm{L})$ consisted of 10 $\mu \mathrm{L} 2 \times$ TB Green Premix Ex Taq II, $0.8 \mu \mathrm{L}$ each primer (10 $\mu \mathrm{M}), 2 \mu \mathrm{L}$ cDNA template (10 ng), $0.4 \mu \mathrm{L}$ ROX Reference Dye $(50 \times)$, and $6 \mu \mathrm{L}$ sterile water. qPCR was performed as follows: an initial step at $95^{\circ} \mathrm{C}$ for $30 \mathrm{~s}$, followed by 40 cycles at $95^{\circ} \mathrm{C}$ for $5 \mathrm{~s}$ and $60^{\circ} \mathrm{C}$ for $30 \mathrm{~s}$. Each sample was amplified in triplicate. Data were analyzed by the $2^{-\Delta \Delta C t}$ relative quantification method.

\section{Western blot analysis}

Zebrafish embryos at $24 \mathrm{hpf}$ were treated with various concentrations of G-Rh2 and $0.1 \%$ DMSO as 
control for $48 \mathrm{~h}$. Embryos were then washed twice with PBS and lysed at $4^{\circ} \mathrm{C}$ in RIPA buffer for $30 \mathrm{~min}$ followed by centrifugation at $13200 \times \mathrm{g}$ for $20 \mathrm{~min}$ at $4^{\circ} \mathrm{C}$. Protein concentration was quantified using the Bradford Protein Assay Kit (Beyotime, China) and equalized before loading. Equal amounts of total protein were mixed with sample buffer and heated to $100^{\circ} \mathrm{C}$ for $5 \mathrm{~min}$. The proteins were separated by $12 \%$ SDS-PAGE and electrotransferred onto polyvinylidene fluoride membranes (Bio-Rad, USA). Blots were blocked with 5\% skim milk in TBST for $3 \mathrm{~h}$ at room temperature and then incubated with zebrafish VEGF monoclonal antibody $(1: 800)$ at $4^{\circ} \mathrm{C}$ overnight. After washing, the membrane was incubated with Mouse IgG horseradish peroxidase conjugated antibody (1:1000) for $1 \mathrm{~h}$ at room temperature. Proteins bands were detected using an ECL advanced western blotting detection kit. $\beta$-actin was used as the loading control. Densitometry quantification was performed using Image $\mathbf{J}$ software (US National Institutes of Health; http://rsb.info.nih.gov/ij/).

Table I. List of primers used for expression analysis.

\begin{tabular}{lll}
\hline $\begin{array}{l}\text { Primer } \\
\text { name }\end{array}$ & Sequence (5'-3') & $\begin{array}{l}\text { Primer } \\
\text { length }\end{array}$ \\
\hline vegf-fw & TGCTCCTGCAAATTCACACAA & 22 \\
$v e g f$-rv & ATCTTGGCTTTTCACATCTGCAA & 24 \\
$f t t 1$-fw & AACTCACAGACCAGTGAACAAGATC & 25 \\
$f t l$-rv & GCCCTGTAACGTGTGCACTAAA & 22 \\
$k d r$-fw & GCAGAATTCATTCCCATGCCGAACATTAC & 29 \\
$k d r$-rv & GTTAAGCTTAGTCTGAGGCGATCTTGAGG & 29 \\
$\beta$-actin-fw & CGAGCAGGAGATGGGAACC & 19 \\
$\beta$-actin-rv & CAACGGAAACGCTCATTGC & 19 \\
\hline
\end{tabular}

\section{Statistical analysis}

Expression analysis and western blot experiments were repeated three times. Data were analyzed using GraphPadPrism 5.0 software (GraphPad Software Inc., San Diego, CA, USA). Statistical significance was assessed one-way analysis of variance and a probability value of $P<0.05$ was considered significant.

\section{RESULTS}

\section{Morphological observations}

Zebrafish embryos at $24 \mathrm{hpf}$ were dechorionated by protease prior to drug treatment (Fig. 1). To determine the optimum drug concentration, embryos were exposed to various concentrations of G-Rh2 ranging from 30-120 $\mu \mathrm{M}$ and morphological changes of the G-Rh2-treated and control embryos at 12, 24 and $48 \mathrm{hpt}$ were examined. The embryos treated with G-Rh2 up to $84.85 \mu \mathrm{M}$ did not show any morphological defects compared with controls, but $\mathrm{G}-\mathrm{Rh} 2$ at $120 \mu \mathrm{M}$ caused morphological changes such as tail bending and pericardial edema, as shown in Figure 2. Therefore, we selected $84.85 \mu \mathrm{M}$ as our maximum treatment concentration in subsequent experiments.
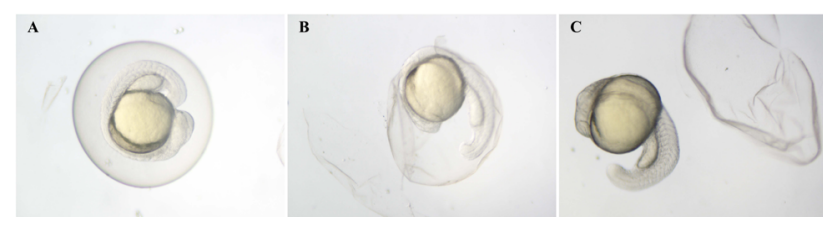

Fig. 1. Dechorionation of zebrafish embryos by protease $(1 \mathrm{mg} / \mathrm{ml})$ at $24 \mathrm{hpf}$. Embryos in the chorion. B. Embryos separating from chorion. C. Embryos out of the chorion. hpf: hours post-fertilization.

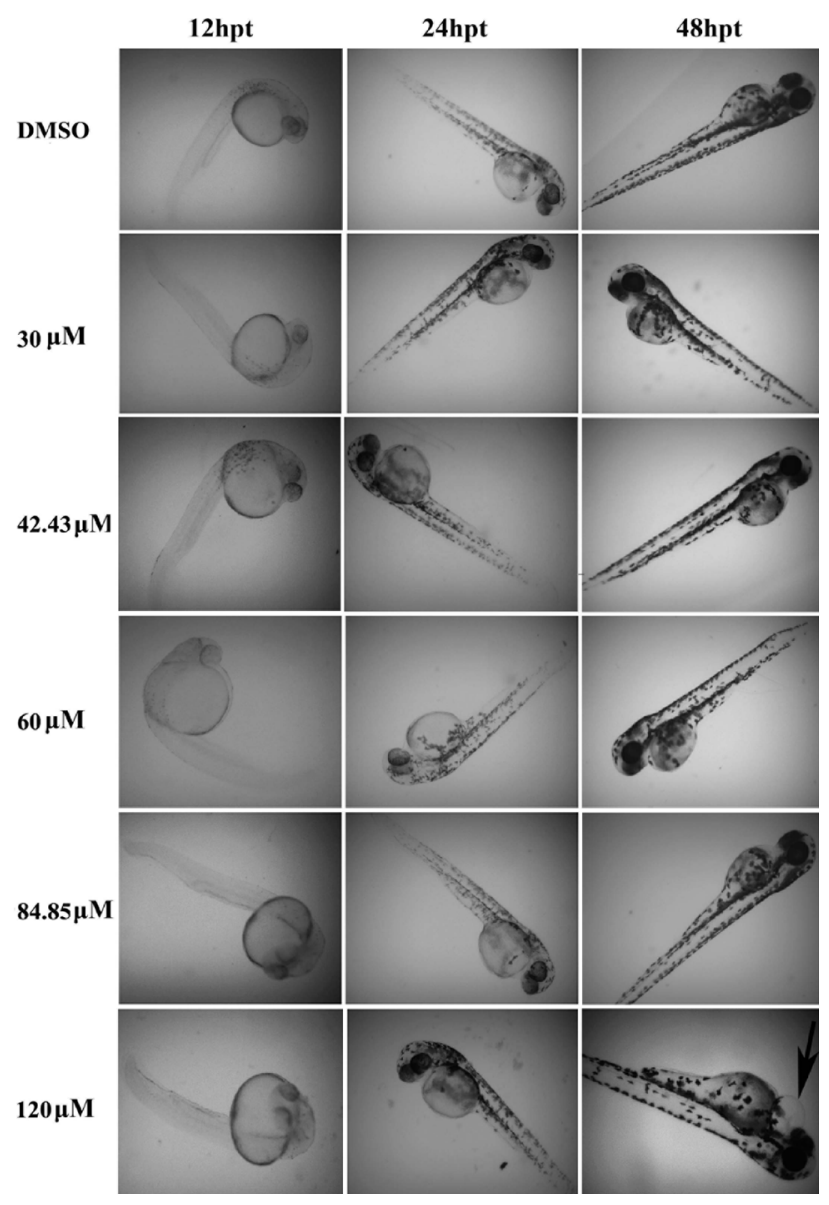

Fig. 2. Morphological changes of zebrafish embryos after G-Rh2 treatment. Zebrafish embryos at $24 \mathrm{hpf}$ were treated with $0.1 \%$ DMSO and various concentrations of G-Rh2 $(30,42.43,60,84.85,120 \mu \mathrm{mol})$ for $48 \mathrm{~h}$. The arrow denotes pericardial edema after treatment with high concentration of G-Rh2 at $120 \mu \mathrm{mol}$. hpt: hours posttreatment. 
Anti-angiogenic activity of $G$-Rh2 in zebrafish

$\operatorname{Tg}(f l i-1: E G F P)$ transgenic zebrafish embryos at 24 hpf were used to evaluate the potential anti-angiogenic activity of G-Rh2. Embryos (24 hpf) were treated with SU5416 (positive control, $5 \mu \mathrm{M}$ ), 0.1\% DMSO (vehicle control), or G-Rh2 $(30,42.43,60$ and $84.85 \mu \mathrm{M})$ for $24 \mathrm{~h}$ and then examined at $48 \mathrm{hpf}$ by fluorescence microscopy. As shown in Figure 3, ISV angiogenesis was most easily observed in the embryos at $48 \mathrm{hpf}$. Treatment with $0.1 \%$ DMSO had no effect on ISV formation (Fig. 3A and A'). SU5416 treatment showed remarkable suppression of ISV formation (Fig. 3B and B'). G-Rh2 inhibited ISV development in zebrafish embryos in a dose-dependent manner compared with the control. The anti-angiogenic effect of G-Rh2 was seen at concentrations ranging from 42.43 to $84.85 \mu \mathrm{M}$ (Fig. 3D-F and $\mathrm{D}^{\prime}-\mathrm{F}^{\prime}$ ). At the concentration of $84.85 \mu \mathrm{M}$ G-Rh2, ISV development was almost completely suppressed (Fig. 3F and $\mathrm{F}^{\prime}$ ).

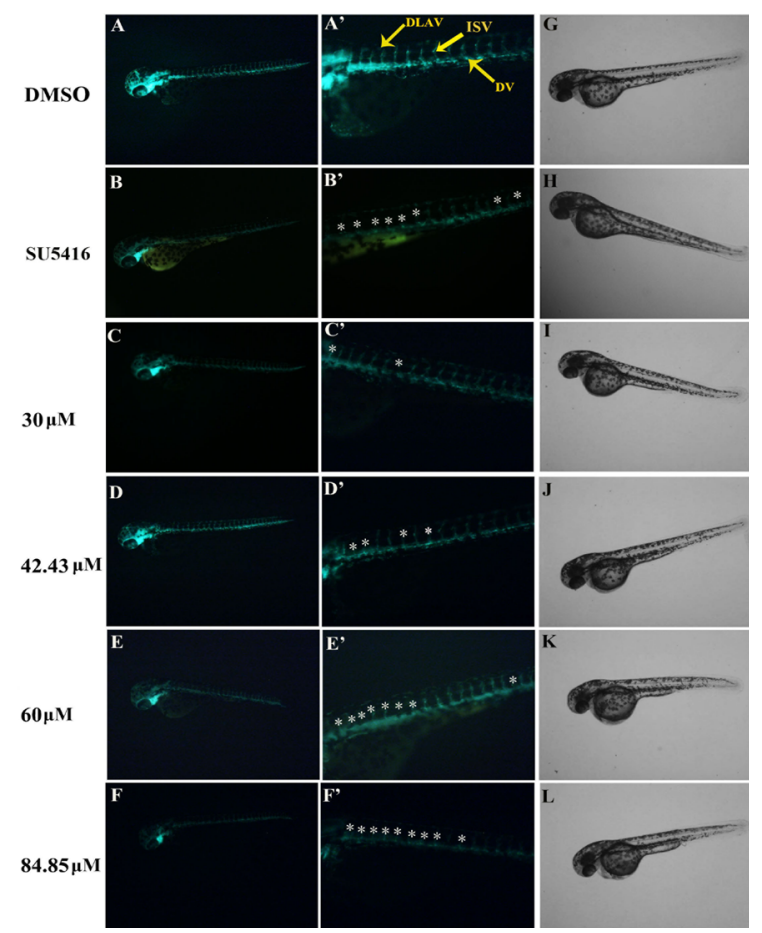

Fig. 3. Anti-angiogenic effect of G-Rh2 on Tg (Fli1$E G F P)$ zebrafish embryos. $24 \mathrm{hpf}$ embryos were treated for $12 \mathrm{~h}$ with (A) $0.1 \%$ DMSO (vehicle control); (B) $5 \mu \mathrm{M}$ SU5416 (positive control); (C) $30 \mu \mathrm{M} \mathrm{G-Rh2;} \mathrm{(D)} 42.43$ $\mu \mathrm{M}$ G-Rh2; (E) $60 \mu \mathrm{M}$ G-Rh2; or (F) $84.85 \mu \mathrm{M}$ G-Rh2. Magnified views of A-F are shown in $\mathrm{A}^{\prime}-\mathrm{F}^{\prime}$. Morphological observation of zebrafish embryos is shown in G-L. The dorsal longitudinal anastomotic vessel (DLAV), dorsal aorta (DA) and intersegmental vessels (ISVs) of zebrafish embryos are indicated by the yellow arrows. Defects in ISV formation are indicated by the white asterisk. $q P C R$

The VEGF signaling pathway plays an important role in angiogenesis and is well studied in zebrafish vascular development (Li et al., 2012; He et al., 2014; Karthik et al., 2014). Vegf is the most studied factor in zebrafish angiogenesis. VEGF receptors are highly specific for VEGF and are markedly upregulated during periods of tumor growth (Cross et al., 2003). To determine the molecular mechanism of G-Rh2-mediated anti-angiogenesis in zebrafish, we examined the gene expressions of vegf and its receptor VEGFR-1 (ftt-l) and VEGFR-2 $(k d r)$ as representatives of the VEGF signaling pathway. As shown in Figure 4, G-Rh2 treatment resulted in significantly decreased vegf mRNA expression in a dose-dependent manner compared with controls (Fig. 4A). However, the expression levels of $f t t 1$ and $k d r$ mRNA were not significantly altered by G-Rh2 treatment (Fig. 4B-C).
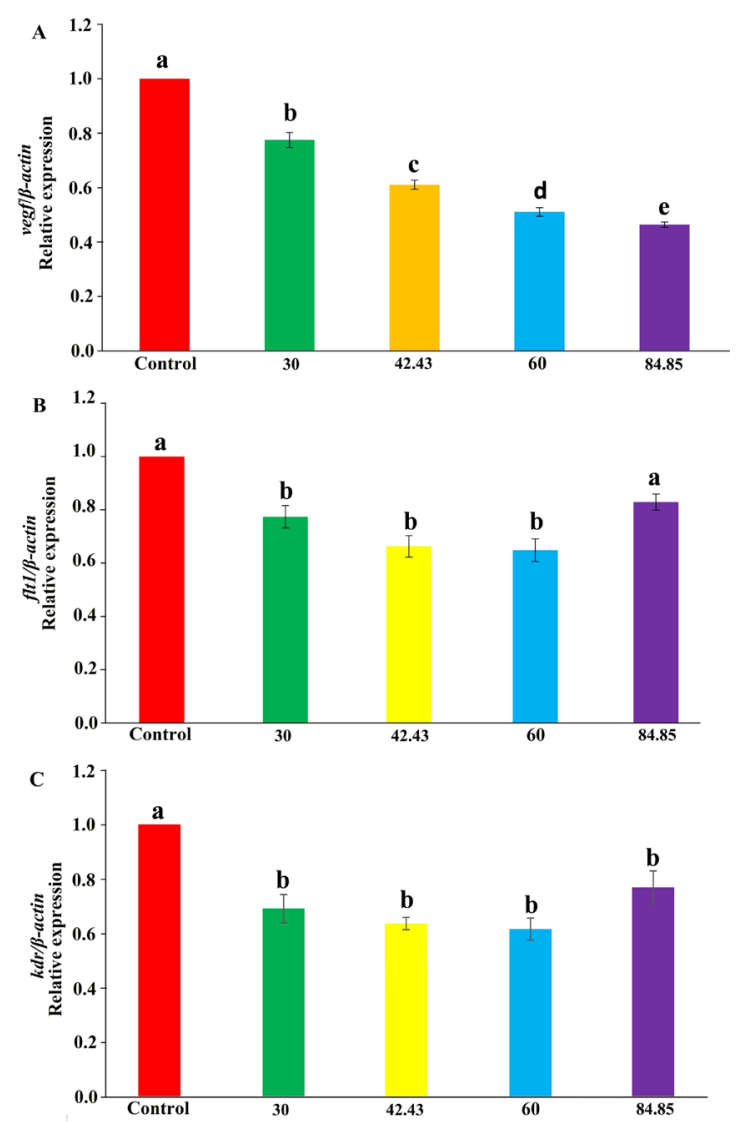

Fig. 4. qPCR analysis of VEGF signaling pathway genes in zebrafish embryos treated with G-Rh2. qPCR analysis of vegf (A), $f t 1$ (B) and $k d r$ (C) mRNA expression in 72 hpf zebrafish embryos treated with different concentrations of G-Rh2 or $0.1 \%$ DMSO (vehicle control). Data are shown as mean \pm SEM of triplicate experiments. Different letters $(a-e)$ above the columns indicate significant differences $(P<0.05)$. 
Western blot analysis

We further analyzed Vegf protein expression in G-Rh2-treated and control zebrafish embryos by western blot analyses. The results showed that Vegf protein expression level was substantially decreased in response to G-Rh2 treatment compared with the control (Fig. 5). These data suggested that G-Rh2 may inhibit angiogenesis by downregulation of Vegf expression in zebrafish embryos.

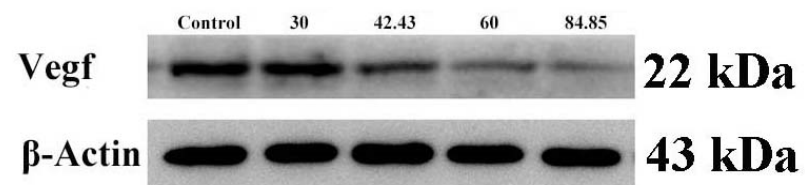

A

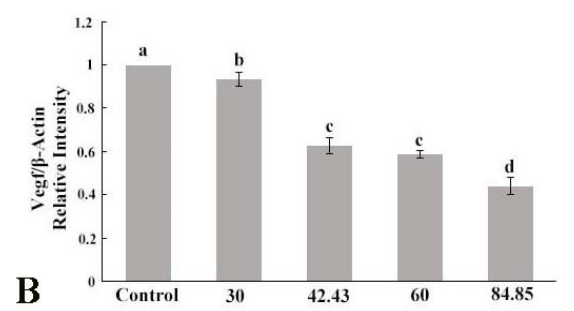

Fig. 5. Western blot analysis of VEGF protein expression in G-Rh2 treated embryos. Western blot of Vegf expression in 72 hpf zebrafish embryos treated with different concentrations of G-Rh 2 or $0.1 \%$ DMSO (vehicle control). $\beta$-Actin was used as loading control. Different letters $(\mathrm{a}-\mathrm{d})$ above the columns indicate significant differences $(\mathrm{P}<$ $0.05)$.

\section{DISCUSSION}

Angiogenesis plays an important role in tumor development, progression, and metastasis (Mantovani, 2010), and antiangiogenic cancer therapy strategies have been extensively studied (Folkman, 1971; Jansen et al., 2004). G-Rh2 is natural glycoside extracted from the traditional medicinal plant red ginseng. Previous in vitro studies indicated that G-Rh2 shows low toxicity, with only a few side effects, and G-Rh2 has been considered as a nutrient with anticancer properties (Zeng and Tu, 2003). G-Rh2 exhibits cytotoxic and apoptotic effects in hepatoma and leukemia Reh cells (Park et al., 2012; Xia et al., 2014). G-Rh2 was also shown to inhibit the tumor cell proliferation and invasiveness in glioma and prostatic cancer (Wu et al., 2011; Zhang et al., 2015; Li et al., 2018) and exhibit anti-cancer activity in human colorectal cancer cells (Han et al., 2016). In addition, G-Rh2 displayed a suppressive effect on tumor growth in nude mice bearing tumors derived from human ovarian cancer cells (Nakata et al., 1998). G-Rh2 also showed antiangiogenesis in a Lewis pulmonary carcinoma mouse model (Cui and Qu, 2011). Therefore, study of the antitumor mechanism of G-Rh2 has become a hotspot in anti-cancer traditional Chinese medicine research. Here we explored the antiangiogenic activity and molecular mechanism of G-Rh2 in zebrafish embryos.

Zebrafish embryos have been widely used as a live in vivo vertebrate model for studying inhibitors of angiogenesis. Zebrafish embryos are especially suitable for vascular development analysis because of their small size, transparency, ease of manipulation and rapid development (Santoro, 2014). Moreover, zebrafish embryos can survive for a week without a blood circulation or vascular system (Li et al., 2016). Many anti-angiogenic drugs tested in this model demonstrated similar responses to those observed in mammalian systems (Cross et al., 2003; Langheinrich, 2003). Angiogenesis is a critical process involved in tumor growth, development and metastasis. The formation of ISVs in zebrafish embryos is a paradigm for studying angiogenesis in vivo (Karthik et al., 2014). Transgenic zebrafish expressing EGFP in blood vessels allow for observation of angiogenesis in live embryos under a fluorescence microscope (Tran et al., 2007). We used the $\mathrm{Tg}(f i 1-E G F P)$ transgenic zebrafish, which express EGFP in endothelial cells, to study anti-angiogenic activity of G-Rh2. We treated zebrafish embryos with G-Rh2 at 24 hpf when the sprouting of ISVs from the vasculogenic vessel and posterior cardinal vein initiated (Letamendia et al., 2012). We did not observe any morphological changes in embryos treated with G-Rh2 up to 84.85 $\mu \mathrm{M}$ but higher concentrations at $120 \mu \mathrm{M}$ caused tail bending and pericardial edema at $72 \mathrm{hpf}$. Moreover, the antiangiogenic effect of G-Rh2 was observed from 42.43 to $84.85 \mu \mathrm{M}$, with a significantly reduced number of ISVs with increasing concentration of the drug. These results indicate that the optimal concentration of G-Rh2 was $84.85 \mu \mathrm{M}$, while concentrations of $120 \mu \mathrm{M}$ or above were toxic to the zebrafish embryos.

VEGF signaling plays a key role in the process of angiogenesis (Ferrara et al., 2003). VEGF is currently the mostly studied growth factor in zebrafish angiogenesis. (Paramasivam et al., 2012). Paramasivam et al. (2012) showed that thymoquinone has potent antiangiogenic activity by downregulating VEGF in the zebrafish model. Karthik et al. (2014) reported that syringic acid can inhibit the formation of blood vessels by downregulation of VEGFmediated angiogenesis in zebrafish embryos. Currently, three VEGF receptors have been found in zebrafish, namely VEGFR1, VEGFR2 and VEGFR3 (Bussmann et al., 2008). He et al. (2014) found that auranofin suppressed VEGF signaling pathway genes (vegf, ftt $1, k d r$ ) but had no impact on thioredoxin reductase in zebrafish. In our study, 
we selected VEGFR-1 ( $f t l)$ and VEGFR-2 $(k d r)$, as these two receptors are expressed mainly in vascular endothelial cells (Goishi and Klagsbrun, 2004). qPCR showed that G-Rh2 only downregulated vegf mRNA levels but did not impact gene expression of its receptors $(f t 1, k d r)$ in zebrafish. We further examined the protein expression of Vegf in zebrafish embryos using western blot analysis and found that G-Rh2 also reduced Vegf expression in zebrafish embryos in a dose-dependent manner. Together these results demonstrate that G-RH2 downregulates Vegf expression in the zebrafish model.

In conclusion, our study suggested for the first time that G-Rh2 inhibits blood vessel formation in zebrafish. We have also shown that the molecular mechanism of the anti-angiogenic activity of G-Rh2 may be due to inhibition of Vegf in zebrafish. This study provides a theoretical basis for further elucidation of the anti-tumor mechanism of G-Rh2 and demonstrated the applicability and feasibility of screening the antiangiogenic activity of natural compounds in the zebrafish model.

\section{ACKNOWLEDGMENTS}

Work was supported by the Experimental Animal Science and Technology Project of Zhejiang Province (grant numbers 2017C37154) and Taizhou Science and Technology Plan Project (grant numbers 15ny11). We thank Edanz Group (www.edanzediting.com/ac) for editing a draft of this manuscript.

\section{Statement of conflict of interest}

The authors have declared no conflict of interest.

\section{REFERENCES}

Berthod, F., 2014. Fibroblasts and endothelial cells: The basic angiogenic unit. Nov. Sci. Publ. Inc., 7: $145-153$.

Bussmann, J., Lawson, N., Zon, L., Schulte-Merker, S., Ekker, M., Mullins, M., Postlethwait, J. and Westerfield, M., 2008. Zebrafish VEGF receptors: A guideline to nomenclature. PLoS Genet., 4: e1000064. https://doi.org/10.1371/journal. pgen. 1000064

Crawford, Y. and Ferrara, N., 2009. VEGF inhibition: insights from preclinical and clinical studies. Cell Tissue Res., 335: 261-269. https://doi.org/10.1007/ s00441-008-0675-8

Cross, L.M., Cook, M.A., Lin, S., Chen, J.N. and Rubinstein, A.L., 2003. Rapid analysis of angiogenesis drugs in a live fluorescent zebrafish assay. Arterioscler. Thromb. Vasc.
Biol., 23: 911-912. https://doi.org/10.1161/01. ATV.0000068685.72914.7E

Cui, Y. and Qu, F., 2011. Antitumor effect of ginsenoside $\mathrm{Rh} 2$ on lewis pulmonary carcinoma bearing in mice. Anti-Tumor Pharm., 1: 422-426.

Dong, X., Han, Z.C. and Yang, R., 2007. Angiogenesis and antiangiogenic therapy in hematologic malignancies. Crit. Rev. Oncol. Hematol., 62: 105-118. https://doi.org/10.1016/j. critrevonc.2006.11.006

Ferrara, N., Gerber, H.P. and LeCouter, J., 2003. The biology of VEGF and its receptors. Nat. Med., 9: 669-676. https://doi.org/10.1038/nm0603-669

Folkman, J., 1971. Tumor angiogenesis: Therapeutic implications. N. Engl. J. Med., 285: 1182-1186. https://doi.org/10.1056/NEJM197111182852108

Goishi, K. and Klagsbrun, M., 2004. Vascular endothelial growth factor and its receptors in embryonic zebrafish blood vessel development. Curr. Top. Dev. Biol., 62: 127-152. https://doi. org/10.1016/S0070-2153(04)62005-9

Han, S., Jeong, A.J., Yang, H., Bin Kang, K., Lee, H., Yi, E.H., Kim, B.H., Cho, C.H., Chung, J.W., Sung, S.H. and Ye, S.K., 2016. Ginsenoside 20(S)-Rh2 exerts anti-cancer activity through targeting IL-6induced JAK2/STAT3 pathway in human colorectal cancer cells. J. Ethnopharmacol., 194: 83-90. https://doi.org/10.1016/j.jep.2016.08.039

He, M.F., Gao, X.P., Li, S.C., He, Z.H., Chen, N., Wang, Y. Bin, and She, J.X., 2014. Anti-angiogenic effect of auranofin on HUVECs in vitro and zebrafish in vivo. Eur. J. Pharmacol., 740: 240-247. https://doi. org/10.1016/j.ejphar.2014.07.034

Huang, Y., Huang, H., Han, Z., Li, W., Mai, Z. and Yuan, R., 2019. Ginsenoside Rh2 inhibits angiogenesis in prostate cancer by targeting CNNM1. $J$. Nanosci. Nanotechnol., 19: 1942-1950. https://doi. org/10.1166/jnn.2019.16404

Jain, R.K., 2005. Antiangiogenic therapy for cancer: current and emerging concepts. Oncology (Williston Park), 19: 7-16.

Jain, R.K., Duda, D.G., Clark, J.W. and Loeffler, J.S., 2006. Lessons from phase III clinical trials on antiVEGF therapy for cancer. Nat. Clin. Pract. Oncol., 3: 24-40. https://doi.org/10.1038/ncponc0403

Jansen, M., De Witt Hamer, P.C., Witmer, A.N., Troost, D. and Van Noorden, C.J.F., 2004. Current perspectives on antiangiogenesis strategies in the treatment of malignant gliomas. Brain Res. Rev., 45: 143-163. https://doi.org/10.1016/j. brainresrev.2004.03.001

Jin, X., Che, D.B., Zhang, Z.H., Yan, H.M., Jia, Z.Y., 
Jia, X. and Bin, 2016. Ginseng consumption and risk of cancer: A meta-analysis. J. Ginseng Res., 40: 269-277. https://doi.org/10.1016/j.jgr.2015.08.007

Karthik, G., Angappan, M., VijayaKumar, A. and Natarajapillai, S., 2014. Syringic acid exerts antiangiogenic activity by downregulation of VEGF in zebrafish embryos. Biomed. Prev. Nutr., 4: 203208. https://doi.org/10.1016/j.bionut.2014.01.007

Kondo, T., Ohta, T., Igura, K., Hara, Y. and Kaji, K., 2002. Tea catechins inhibit angiogenesis in vitro, measured by human endothelial cell growth, migration and tube formation, through inhibition of VEGF receptor binding. Cancer Lett., 180: 139-144. https://doi.org/10.1016/S03043835(02)00007-1

Langheinrich, U., 2003. Zebrafish: A new model on the pharmaceutical catwalk. BioEssays, 25: 904-912. https://doi.org/10.1002/bies.10326

Letamendia, A., Quevedo, C., Ibarbia, I., Virto, J.M., Holgado, O., Diez, M., Izpisua Belmonte, J.C. and Callol-Massot, C., 2012. Development and validation of an automated high-throughput system for zebrafish in vivo screenings. PLoS One, 7: e36690. https://doi.org/10.1371/journal. pone. 0036690

Li, D., Li, Xiang Pen, Wang, H.X., Shen, Q.Y., Li, Xiang Ping, Wen, L., Qin, X.J., Jia, Q.L., Kung, H.F. and Peng, Y., 2012. VEGF induces angiogenesis in a zebrafish embryo glioma model established by transplantation of human glioma cells. Oncol. Rep., 28: 937-942. https://doi.org/10.3892/or.2012.1861

Li, J., Peng, Y., Li, S., Sun, Y., Chan, J.Y.W., Cui, G., Wang, D., Zhou, G.C. and Lee, S.M.Y., 2016. Anti-angiogenic activity of a new andrographolide derivative in zebrafish and HUVECs. Eur. J. Pharmacol., 789: 344-353. https://doi. org/10.1016/j.ejphar.2016.07.043

Li, K.F., Kang, C.M., Yin, X.F., Li, H.X., Chen, Z.Y., Li, Y., Zhang, Q. and Qiu, Y.R., 2018. Ginsenoside Rh2 inhibits human A172 glioma cell proliferation and induces cell cycle arrest status via modulating Akt signaling pathway. Mol. Med. Rep., 17: 30623068. https://doi.org/10.3892/mmr.2017.8193

Mantovani, A., 2010. Molecular pathways linking inflammation and cancer. Curr. mol. Med., 10: 369373. https://doi.org/10.2174/156652410791316968

Matulonis, U.A., Berlin, S., Ivy, P., Tyburski, K., Krasner, C., Zarwan, C., Berkenblit, A., Campos, S., Horowitz, N., Cannistra, S.A., Lee, H., Lee, J., Roche, M., Hill, M., Whalen, C., Sullivan, L., Tran, C., Humphreys, B.D. and Penson, R.T., 2009. Cediranib, an oral inhibitor of vascular endothelial growth factor receptor kinases, is an active drug in recurrent epithelial ovarian, fallopian tube, and peritoneal cancer. J. clin. Oncol., 27: 5601-5606. https://doi.org/10.1200/JCO.2009.23.2777

Nakata, H., Kikuchi, Y., Tode, T., Hirata, J., Kita, T., Ishii, K., Kudoh, K., Nagata, I. and Shinomiya, N., 1998. Inhibitory effects of ginsenoside Rh2 on tumor growth in nude mice bearing human ovarian cancer cells. Japanese J. Cancer Res., 89: 733740. https://doi.org/10.1111/j.1349-7006.1998. tb03278.x

Natale, R.B., Bodkin, D., Govindan, R., Sleckman, B.G., Rizvi, N.A., Capó, A., Germonpré, P., Eberhardt, W.E.E., Stockman, P.K., Kennedy, S.J. and Ranson, M., 2009. Vandetanib versus gefitinib in patients with advanced non-small-cell lung cancer: Results from a two-part, double-blind, randomized phase II study. J. clin. Oncol., 27: 2523-2529. https://doi. org/10.1200/JCO.2008.18.6015

Paramasivam, A., Kalaimangai, M., Sambantham, S., Anandan, B. and Jayaraman, G., 2012. Antiangiogenic activity of thymoquinone by the downregulation of VEGF using zebrafish (Danio rerio) model. Biomed. Prev. Nutr., 2: 169-173. https:// doi.org/10.1016/j.bionut.2012.03.011

Park, H.M., Kim, S.J., Kim, J.S. and Kang, H.S., 2012. Reactive oxygen species mediated ginsenoside $\mathrm{Rg} 3$ - and Rh2-induced apoptosis in hepatoma cells through mitochondrial signaling pathways. Fd chem. Toxicol., 50: 2736-2741. https://doi. org/10.1016/j.fct.2012.05.027

Saltz, L.B., Clarke, S., Díaz-Rubio, E., Scheithauer, W., Figer, A., Wong, R., Koski, S., Lichinitser, M., Yang, T.S., Rivera, F., Couture, F., Sirzén, F. and Cassidy, J., 2008. Bevacizumab in combination with oxaliplatin-based chemotherapy as first-line therapy in metastatic colorectal cancer: A randomized phase III study. J. clin. Oncol., 26: 2013-2019. https:// doi.org/10.1200/JCO.2007.14.9930

Sandler, A., Gray, R., Perry, M.C., Brahmer, J., Schiller, J.H., Dowlati, A., Lilenbaum, R. and Johnson, D.H., 2006. Paclitaxel-carboplatin alone or with bevacizumab for non-small-cell lung cancer. N. Engl. J. Med., 355: 2542-2550. https://doi. org/10.1056/NEJMoa061884

Santoro, M.M., 2014. Antiangiogenic cancer drug using the zebrafish model. Arterioscler. Thromb. Vasc. Biol., 34: 1846-1853. https://doi.org/10.1161/ ATVBAHA.114.303221

Shi, Q., Li, J., Feng, Z., Zhao, L., Luo, L., You, Z., Li, D., Xia, J., Zuo, G. and Chen, D., 2014. Effect of ginsenoside Rh2 on the migratory ability of HepG2 
liver carcinoma cells: Recruiting histone deacetylase and inhibiting activator protein 1 transcription factors. Mol. Med. Rep., 10: 1779-1785. https://doi. org/10.3892/mmr.2014.2392

Thisse, C. and Zon, L.I., 2002. Organogenesis - heart and blood formation from the zebrafish point of view. Science, 295: 457-462. https://doi.org/10.1126/ science. 1063654

Tran, T.C., Sneed, B., Haider, J., Blavo, D., White, A., Aiyejorun, T., Baranowski, T.C., Rubinstein, A.L., Doan, T.N., Dingledine, R. and Sandberg, E.M., 2007. Automated, quantitative screening assay for antiangiogenic compounds using transgenic zebrafish. Cancer Res., 67: 11386-11392. https:// doi.org/10.1158/0008-5472.CAN-07-3126

Westerfield, M., 2000. The zebrafish book: A guide for the laboratory use of zebrafish (Brachydanio rerio). $4^{\text {th }}$ edn. Univ. of Oregon Press, Eugene.

Wong, M.L., Prawira, A., Kaye, A.H. and Hovens, C.M., 2009. Tumour angiogenesis: Its mechanism and therapeutic implications in malignant gliomas. J. clin. Neurosci., 16: 1119-1130. https://doi. org/10.1016/j.jocn.2009.02.009

Wu, N., Wu, G.C., Hu, R., Li, M. and Feng, H., 2011. Ginsenoside Rh2 inhibits glioma cell proliferation by targeting microRNA-128. Acta Pharmacol. Sin., 32: 345-353. https://doi.org/10.1038/aps.2010.220

Xia, T., Wang, J.-C., Xu, W., Xu, L.-H., Lao, C.-H., Ye, Q.-X. and Fang, J.-P., 2014. 20(S)-ginsenoside Rh2 induces apoptosis in human leukaemia reh cells through mitochondrial signaling pathways. Biol. Pharm. Bull., 37: 248-254. https://doi.org/10.1248/ bpb.b13-00667

Yang, J.C., Haworth, L., Sherry, R.M., Hwu, P., Schwartzentruber, D.J., Topalian, S.L., Steinberg, S.M., Chen, H.X. and Rosenberg, S.A., 2003. A randomized trial of bevacizumab, an anti-vascular endothelial growth factor antibody, for metastatic renal cancer. N. Engl. J. Med., 349: 427-434. https:// doi.org/10.1056/NEJMoa021491

Zeng, X.L. and Tu, Z.G., 2003. In vitro induction of differentiation by ginsenoside Rh2 in SMMC-7721 hepatocarcinoma cell line. Pharmacol. Toxicol., 93: 275-283. https://doi.org/10.1111/j.1600-0773.2003. pto930605.X

Zhang, Q., Hong, B., Wu, S. and Niu, T., 2015. Inhibition of prostatic cancer growth by ginsenoside $\mathrm{Rh} 2$. Tumor Biol., 36: 2377-2381. https://doi.org/10.1007/ s13277-014-2845-5 